\title{
Características del estudiante mentor en la educación superior
}

\section{Characteristics of the student mentor in higher education \\ Características do aluno mentor no ensino superior}

Fabiola Lydie Rochin Berumen

Universidad Autónoma de Zacatecas, Unidad Académica de Medicina Veterinaria y Zootecnia, México fabiolauaz@outlook.com https://orcid.org/0000-0002-8676-7768

Heriberto Rodríguez Frausto

Universidad Autónoma de Zacatecas, Unidad Académica de Medicina Veterinaria y Zootecnia, México mvzhrf1958@gmail.com https://orcid.org/0000-0001-9608-5843

Francisco Javier Gutiérrez Piña Universidad Autónoma de Zacatecas, Unidad Académica de Medicina Veterinaria y Zootecnia, México

fj_gp@yahoo.com.mx https://orcid.org/0000-0001-5743-254X

Juan Antonio Ramírez Chequer

Universidad Autónoma de Zacatecas, Unidad Académica de Medicina Veterinaria y Zootecnia, México Chequer-r@hotmail.com https://orcid.org/0000-0001-6400-0390 


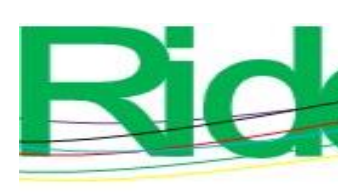

Revista Iberoamericana para la Investigación y el Desarrollo Educativo ISSN 2007 - 7467

\title{
Resumen
}

El plan de estudios del programa de licenciatura de Medicina Veterinaria y Zootecnia de la Universidad Autónoma de Zacatecas (UAZ) es un programa de calidad, con reconocimiento nacional e internacional, donde uno de los indicadores de calidad más relevantes ante los organismos acreditadores, es mantener una eficiencia termina alta en dicho programa, para lo cual se debe prestar especial atención a los indicadores intermedios de promoción intersemestral, egreso y titulación. Para tal efecto, además de dar cabal cumplimiento a las actividades curriculares formales, también se incorporan las mentorías para evitar la deserción y coadyuvar al cumplimiento de los objetivos mencionados. La mentoría es un programa que lucha por lograr el reconocimiento curricular, donde los estudiantes sobresalientes que apoyan académicamente a compañeros en riesgo de rezago sientan el respaldo institucional, principalmente por parte del cuerpo de profesores. El presente trabajo, por tanto, se enfoca en describir las características de los actores del proceso de mentoría con base en una metodología cualitativa. La literatura revisada respecto al tema se basa en aportaciones teóricas sobre las formas en que se interrelacionan los actores involucrados en la mentoría. Además, se hace un análisis de cómo los modelos educativos deben de retomar las acciones de mentoría para que en el futuro sean reconocidas de manera institucional.

Palabras clave: características, formación académica, mentor, mentoría.

\begin{abstract}
The Study Plan of the Veterinary Medicine and Zootechnics Degree program of the Autonomous University of Zacatecas (UAZ), is a quality program, with national and international recognition, where one of the most relevant quality indicators before the accrediting bodies is maintaining an efficiency ends high in such a program, with national and international recognition, where one of the most relevant quality indicators before the accrediting bodies is maintaining an efficiency ends high in such a program. That is why special attention should be paid to the intermediate indicators of inter-monthly promotion, egress, and graduation. For this purpose, in addition to fully complying with the formal curricular activities, mentoring activities are also incorporated to avoid dropping out and contribute to the fulfillment of the aforementioned objectives. Mentoring is a program that strives to achieve curricular recognition, where outstanding students who academically support peers at risk of lagging, feel institutional support mainly from the faculty. This work
\end{abstract}




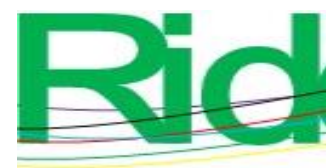

Revista Iberoamericana para la
Investigación y el Desarrollo Educativo
ISSN $2007-7467$

addresses an analysis of the characteristics of the actors in the mentoring process, under a qualitative development methodology. The literature that is analyzed on the subject is based on theoretical contributions on how the actors involved in mentoring interrelate; In the same way, an analysis is made of how educational models should take up the mentoring actions to pay for them to be recognized institutionally in the future.

Keywords: characteristics, academic training, mentoring, mentoring.

\section{Resumo}

O plano de estudos do programa de licenciatura em Medicina Veterinária e Zootecnia da Universidade Autónoma de Zacatecas (UAZ) é um programa de qualidade, com reconhecimento nacional e internacional, onde um dos indicadores de qualidade mais relevantes perante os organismos credenciadores é manter uma eficiência fins elevados no referido programa, para o qual deve ser dada atenção especial aos indicadores intermediários de promoção, graduação e pós-graduação mensais. Para tanto, além do cumprimento integral das atividades curriculares formais, também é incorporada a tutoria para evitar o abandono escolar e contribuir para o cumprimento dos objetivos acima mencionados. O Mentoring é um programa que visa o reconhecimento curricular, onde os alunos que se destacam, que apoiam academicamente os pares em risco de atraso, sentem o apoio institucional, principalmente do corpo docente. O presente trabalho, portanto, se concentra em descrever as características dos atores do processo de mentoria com base em uma metodologia qualitativa. A literatura revisada sobre o assunto fundamenta-se em contribuições teóricas sobre as formas como os atores envolvidos na mentoria se relacionam. Além disso, é feita uma análise de como os modelos educacionais devem retomar as ações de mentoria para que, no futuro, sejam reconhecidos institucionalmente.

Palavras-chave: características, formação acadêmica, mentor, mentoria.

Fecha Recepción: Julio 2021

Fecha Aceptación: Enero 2022 


\section{Revista Iberoamericana para la Investigación y el Desarrollo Educativo ISSN 2007 - 7467}

\section{Introducción}

El Centro Académico de Servicios Escolares (CASE) de la Universidad Autónoma de Zacatecas (UAZ) en conjunto con la Unidad Académica de Medicina Veterinaria y Zootecnia (UAMVZ) dan seguimiento al Programa de Mentorías (creado en 2015) para orientar a los alumnos de nuevo ingreso, por lo que en este trabajo se analizan los procedimientos llevados a cabo en él para brindar propuestas que permitan mejorarlo. Los estudiantes que han participado en dicho programa manifiestan que es de gran utilidad y una buena estrategia de apoyo para el avance, especialmente de alumnos de nuevo ingreso, quienes más dificultades enfrentan al inicio de su formación profesional. En tal sentido, la coordinación de mentoría de la UAMVZ cuenta con procedimientos sistematizados para que los estudiantes mentores realicen las actividades de una manera planificada y organizada, donde se brinde apoyo académico al alumno de nuevo ingreso. Para ello, el estudiante (mentor) debe poseer ciertas características, las cuales es necesario reconocer para brindar un servicio óptimo que evite la deserción escolar.

De acuerdo con Clutterbuck et al. (2017), la actividad de mentoría se remonta a siglos, como una forma de interacción entre los involucrados de acuerdo a las características de desarrollo social. En la actualidad, los programas académicos, el contexto, las necesidades individuales y de grupo y la relación social en que se desenvuelven marcan la pauta de cómo llevar a cabo los programas de mentorías (Orland Barak, 2016). Ese acompañamiento se ha convertido en una herramienta indispensable durante el ingreso, estancia y egreso de los estudiantes, por lo que debe ser en una forma de vida académica. En efecto, la mentoría se convierte en baluarte cuando los alumnos de grados avanzados, voluntarios y sobresalientes facilitan ese tránsito mediante un programa formal de convivencia y de reflexión acerca de las acciones y conceptos para la construcción del conocimiento (Alonso et al., 2012).

El programa de mentoría - que en la mayoría de las instituciones de educación superior aún es considerado como informal, ya que no figura dentro de las actividades académicas sustantivas - permite a los alumnos obtener resultados académicos y vocacionales positivos (van Dam et al., 2018). Tradicionalmente, la mentoría ha sido

considerada como una relación informal entre personas mayores o con mayor avance académico, quienes logran empatía con personas o alumnos jóvenes de una organización (Garringer et al., 2015). 


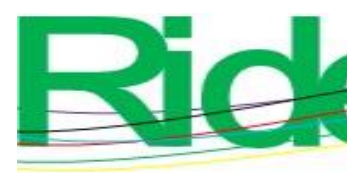

Revista Iberoamericana para la
Investigación y el Desarrollo Educativo
ISSN $2007-7467$

Ahora bien, tomando como base la experiencia docente y la contribución a la formación de estudiantes mentores, los autores del presente trabajo consideran a la mentoría como un aprendizaje incidental e informal. Dicha experiencia se ha registrado en el área de cirugía veterinaria, donde los alumnos sobresalientes apoyan a quienes se les dificulta el proceso quirúrgico en animales vivos debido a diversos factores, como estrés, miedo, etc. Esta forma de ubicar mentores y mentorados se ubica en un programa informal donde los estudiantes se brindan la confianza y establecen su rol correspondiente. Se crea un clima diferente al que se da en el escenario formal entre estudiante y el docente, y se aprende a descubrir la realidad en la que están inmersos. El mentor también aprende al observar y conocer a su discípulo y amigo desde la perspectiva de este, ya que subyacen aspectos culturales y de otra índole que lo ubicaron ahí. Por ello, en la mentoría lo central no es tan solo lograr metas, sino el proceso que se vive.

\section{Conceptualización sobre la mentoría}

Se conoce que la mentoría tiene sus orígenes en la mitología griega, específicamente en la Odisea de Homero, donde Mentor era el profesor de Telémaco, hijo de Ulises. Ante la ausencia temporal de la figura paterna debido a cuestiones bélicas, surge en el terreno educativo el papel del tutor. El nivel de participación se daba al grado tal que el mentor era considerado mitad dios y mitad hombre, y desempeñaba papeles propios del hombre como de la mujer. De hecho, asumía un rol preponderante que correspondía a los padres de familia con un fuerte sentido de credibilidad y sabiduría (Díaz y Bastías, 2013).

En la actualidad, la mentoría la ejecutan alumnos, identificados y entrenados por sus cualidades personales y académicas, para apoyar a otros alumnos. Las mentorías son individuales o grupales para solucionar un problema, completar una tarea, aprender alguna estrategia, dominar un procedimiento, etc., por lo que sirve para apoyar la función de los profesores (Quevedo Marcolino y Medeiros Rodrigues Reali, 2010).

Para Gómez y Eisman (2001), la mentoría grupal identifica problemas de orden escolar, personal, familiar o social que afectan el proceso formativo, y canaliza a instancias internas para su debido cuidado. En cambio, la mentoría individualizada se encarga de aquellos aspectos relacionados con la pedagogía y los métodos de enseñanza con el propósito de que el aprendizaje fluya a la par del desarrollo de valores, actitudes, destrezas y competencias para una formación más integral en lo profesional y humano del individuo mentorado. 


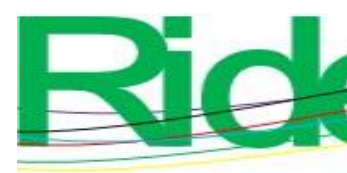

Revista Iberoamericana para la
Investigación y el Desarrollo Educativo
ISSN $2007-7467$

La mentoría, entonces, es una orientación directa al estudiante de acuerdo con el protocolo de mentoría entre iguales, donde se realizan las distintas actividades de evaluación establecidas (Soto et al., 2012). En otras palabras, la mentoría es un tipo de relación en la que el aprendizaje y la experimentación pueden ocurrir, las habilidades potenciales pueden ser desarrolladas y los resultados medidos en términos de competencias ganadas.

Esta actividad tiene cabida en el esquema de supervisión que platea Ten Cate (2018) en su propuesta teórica orientada al desarrollo de las competencias a través del modelo denominado actividades profesionales confiables. En él se establece una pirámide de desarrollo del alumno que inicia en el nivel en que solo se le permite estar de observador de una actividad académica o de servicio profesional realizado por parte de expertos y alumnos avanzados en grado superiores; después, a ese alumno observador le permiten hacer las tareas o actividades conjuntamente con su maestro supervisor. De ese modo se le van confiando actividades hasta que alcanza la independencia y autonomía en sus decisiones para ejecutar con maestría las actividades que le fueron encomendadas bajo su responsabilidad. En ese momento, el alumno que logra escalar dicha pirámide está en condiciones de ser mentor para supervisar a quienes inician el mismo recorrido, pero que manifiestan diferente tipo de situaciones o vacíos que lo hacen candidato para ser apoyado.

Identificadas las definiciones de mentoría y tomando en cuenta que el mentor funge como un partícipe indispensable en ella, el sujeto de la mentoría se define como una persona que está dispuesta a ayudar e invertir tiempo y energía en el proceso de compartir su conocimiento y experiencia. Al asumir esta responsabilidad debe comunicarse con asertividad, proporcionar retroalimentación de forma constructiva, guiar, proveer información de ideas, confrontar, alentar y explorar opciones (Soler, 2005).

La mentoría debe establecerse ante una necesidad, la cual es incitadora de una actividad de intercambio entre dos o más personas. La noción de necesidad — según Heller (1996) - oscila entre los deseos y las carencias. No obstante, saber lo que se necesita es realmente difícil, por lo que el trabajo de los mentores, profesionales y científicos es ayudar a precisarlas en una situación dada.

Concretamente, la actividad de mentoría se enfoca en atender intereses definidos y a resolver necesidades, deseos, expectativas y aun caprichos. Es decir, la definición de una necesidad implica considerar aspectos objetivos y subjetivos, lo que implica perspectivas emocionales y culturales de los destinatarios del servicio ofrecido. Para Miettinen (2005) una 


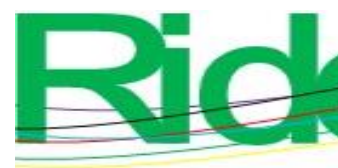

Revista Iberoamericana para la
Investigación y el Desarrollo Educativo
ISSN $2007-7467$

necesidad es entendida como una contradicción o una posibilidad por resolver a través de la actividad mediada por la utilización de herramientas. Para eso, es necesario imaginar al objeto - en este caso el conocimiento ausente en el alumno mentorado - para crear acciones que permitan superar dicho dilema, ya sea creando nuevas alternativas o transformando las existentes, pues al satisfacer necesidades se desarrollan capacidades y competencias individuales y colectivas. Así, la necesidad deviene en el motivo capaz de orientar las acciones del sujeto solo cuando este encuentre a su objeto.

Una de las características de la mentoría en el ámbito universitario es el intercambio de ideas y conceptos, ya que permite la transferencia de conocimientos y experiencias, lo que permite que la relación entre los mentores y mentorados se fortalezca (Burgos, 2018).

\section{Método}

El presente trabajo se sustentó principalmente en el enfoque cualitativo, pues la parte central se basó en una revisión bibliográfica. Entre los principales puntos que sobresalen de dicha revisión destaca la necesidad de una formación continua de los estudiantes mentores, y se rescatan las características que este debe tener para ejercer dicha labor en la educación superior.

En tal sentido, la integridad e independencia en el desarrollo curricular que muestran los estudiantes para gestión del conocimiento y la toma de decisiones parece ser un elemento que los irá perfilando de manera institucional en el grupo de prominentes mentores. En otras palabras, son los alumnos que sobresalen en los aspectos más objetivos del conocimiento, habilidades específicas y actitudes en su disciplina; además, muestran cualidades para la socialización, calidez humana, facilidad para la interacción y el trabajo en equipo, actitudes de liderazgo, así como disposición para llevar de manera paralela y organizada tanto los asuntos propios como los de las personas a su cargo (Escudero, 2017; Terrion y Leonard, 2010). 


\section{Revista Iberoamericana para la Investigación y el Desarrollo Educativo ISSN 2007 - 7467}

\section{Características del estudiante mentor}

Ser alumno de la UAMVZ de la UAZ con promedio mínimo de 8.5 , lo que se constata a través del Kardex, documento oficial del avance académico de los estudiantes de la carrera de Médico Veterinario Zootecnista.

Demostrar experiencia en el área que va a incursionar como mentor, con dominio de procesos y conocimientos de aquellas áreas donde estadísticamente se reporta un mayor índice de reprobación como posible motivo de deserción escolar, o bien en donde se le solicite un apoyo extraordinario por parte de alumnos de grados inferiores o iguales al del mentor.

> Adaptación al ritmo de aprendizaje del estudiante mentorado.

> Tener disponibilidad de tiempo sin descuidar su propio progreso académico.

$>$ Organizar sus actividades para también dedicar espacio a la capacitación continua.

> Manejo de situaciones complicadas. Mostrarse empático con el estudiante mentor y tomar en cuenta la escucha activa para aclarar cualquier necesidad académica que se presente.

$>$ Interés por ayudar a otros. Solidaridad con el estudiante mentor.

$>$ Tener una conducta intachable dentro y fuera de la institución.

$>$ Contar con calificación aprobatoria en todas las materias y no tener ningún examen extraordinario.

$>$ Conocimiento de la institución, de manera especial del reglamento académico sobre el ingreso, permanencia y egreso de la licenciatura en MVZ.

$>$ Carisma y empatía con sus compañeros mentorados. Comprender las situaciones que se viven en el mundo actual por problemas económicos, de salud y de aprendizaje.

$>$ Habilidades interpersonales como liderazgo, capacidad de escucha, trabajo en equipo.

$>$ Contar con valores de compromiso social, honestidad, justicia, democracia, pensamiento crítico-científico y código de ética.

$>$ Discreción ante lo comentado por el alumno mentorado.

$>$ Involucrarse en cursos de inducción de formación de mentores de la UAMVZ. 


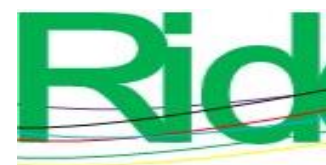

Revista Iberoamericana para la
Investigación y el Desarrollo Educativo
ISSN $2007-7467$

\section{Discusión}

El programa institucional de mentoría de la UAZ en conjunto con la Coordinación de Mentorías de la UAMVZ son los encargados de que los estudiantes mentores sigan las estrategias de apoyo y orientación del programa para asesorar a los alumnos de nuevo ingreso y garantizar así su mejora académica. En este sentido, cabe resaltar que para la elección de los mentores se deben identificar las características aquí establecidas. Una vez seleccionados, deben ser reclutados y capacitados para un mejor desempeño de la tarea encomendada.

\section{Conclusiones}

Los beneficios del programa de mentorías se vinculan con la orientación social y administrativa, hábitos y técnicas de estudio, organización y rendimiento académico, y apoyo en la trayectoria académica.

El diseño del programa institucional de mentoría, por ende, está enfocado en ofrecer servicios académicos a los estudiantes de nuevo ingreso y de semestres avanzados, en caso de ser solicitado. Para ello, quedan establecidas las características que debe poseer el estudiante mentor de educación superior del programa institucional de mentoría de la UAMVZ de la UAZ.

Dentro de las limitantes detectadas está el hecho de que algunos docentes no están de acuerdo con que el programa de mentorías apoye los rezagos que su contenido o metodología no permiten el avance sus estudiantes. Aunado a ello, la época actual de la modalidad de estudiar de manera virtual en ocasiones dificulta la comunicación entre estudiantes para recibir la mentoría.

\section{Futuras líneas de investigación}

En principio, cabe subrayar que se debe fomentar el reconocimiento de los mentores por su contribución a los servicios académicos. Asimismo, se puede profundizar en temas como los siguientes: 1) el rol del estudiante mentor, 2) la mentoría en el nivel superior, 3) la mentoría virtual entre pares, 4) la mentoría como estrategia educativa, 5) los procesos de la mentoría, 6) el impacto social del programa de mentoría, y 7) la mentoría online entre pares debido a la covid-19. 


\section{Referencias}

Alonso, M. A., Calles Doñate, A. y Sánchez Ávila, C. (2012). Diseño y desarrollo de programas de mentoring en organizaciones. Madrid: Síntesis.

Burgos, R. (2018). Modelos de gestión de tutoría según el mentoring para mejorar la formación integral de estudiantes de la Escuela de Administración (tesis de doctorado). Universidad César Vallejo. Recuperado de https://repositorio.ucv.edu.pe/handle/20.500.12692/36521

Clutterbuck, D., Kochan, F., Gail, L., Domínguez, N. and Haddock, J. (ed.) (2017). The SAGE Handbook Of Mentoring. USA: Arrangement. Retrieved from https://n9.cl/t7xs

Díaz, C. y Bastías, C. (2013). Los procesos de la mentoría en la formación inicial docente. Revista Internacional de Investigación en Ciencias Sociales, 9(2), 301-315. Recuperado de http://dialnet.unirioja.es/servlet/articulo?codigo=4714183

Escudero, L. (2017). Entre pares nos acompañamos. Congresos CLABES. Recuperado de https://revistas.utp.ac.pa/index.php/clabes/article/view/1581

Garringer, M., Kupersmidt, J., Rhodes, J., Stelter, R. and Tai, T. (2015). Elements of effective practice for mentoring ( $4^{\text {th }}$ ed.). Boston, MA: MENTOR: The National Mentoring Partnership. Retrieved from http://www.mentoring.org/program-resources/elementsof-effective-practice-for-mentoring

Gómez, J. A. y Eisman, E. M. (2011). La mentoría como elemento de mejora en el proceso de enseñanza-aprendizaje. En Escuela Técnica Superior de Ingeniería Informática (ed.), XVII Jornadas de Enseñanza Universitaria de la Informática (pp. 411-418). Universidad de Sevilla. Recuperado de http://www.sevillacb.com/es/agenda/ver/78?slug=jornadas-de-ensenanzauniversitaria-de-la-informatica-jenui

Heller, A. (1996). Una revisión de la teoría de las necesidades. Barcelona: Paidós.

Miettinen, R. (2005). Object of activity and individual motivation. Mind, Culture, and Activity, 12(1), 52-69.

Orland-Barak, L. (2016). Mentoring. In Loughran, J. and Hamilton, M. L. (eds.), International Handbook of Teacher Education (vol. 2) (pp. 105-141). Doi: https://doi.org/10.1007/978-981-10-0369-1_5 
Quevedo Marcolino, T. y Medeiros Rodrigues Reali, A. M. (2010). El trabajo del mentor: análisis de los feedbacks de diarios reflexivos a lo largo de un proceso de mentoría en grupo. Revista Iberoamericana de Educación, 52(6), 1-13.

Soler, (2005). El mentoring como herramienta de motivación y retención del talento. Capital Humano, (184). Recuperado de http://pdfs.wke.es/2/2/7/6/pd0000012276.pdf

Soto, N. M., Cuadrado, A. M., García, M. S., Rísquez, A. y Ortega, M. S. (2012). El rol del mentor en un proceso de mentoría universitaria. Educación XX1, 15(2).

Ten Cate, O. (2018). Korean J. Med. Educ. An Introduction to trusted Professional Activities, $30(1), 1-10$.

Terrion, J. L. and Leonard, D. (2010). Motivation of paid peer mentors and unpaid peer helpers in higher education. International Journal of Evidence Based Coaching and Mentoring, 8(1), 85-103. Retrieved from https://radar.brookes.ac.uk/radar/items/8c6a37ad-0979-4c02-95f5-80f8071f62e6/1/

Van Dam, L., Smit, D., Wildschut, B., Branje, S. J. T., Rhodes, J. E., Assink, M. and Stams, G. J. J. M. (2018). Does natural mentoring matter? A multilevel meta-analysis on the association between natural mentoring and youth outcomes. American Journal of Community Psychology, 62(1-2), 203-220. 


\begin{tabular}{|c|c|}
\hline 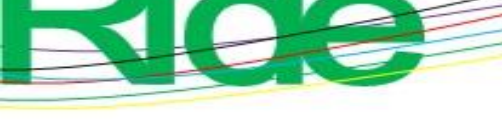 & $\begin{array}{l}\text { Revista lberoamericana para la } \\
\text { Investigación y el Desarrollo Educativo } \\
\text { ISSN } 2007-7467\end{array}$ \\
\hline Rol de Contribución & Autor (es) \\
\hline Conceptualización & Fabiola Lydie Rochin Berumen \\
\hline Metodología & Fabiola Lydie Rochin Berumen; Heriberto Rodríguez Frausto \\
\hline Software & $\begin{array}{l}\text { Fabiola Lydie Rochin Berumen; Heriberto Rodríguez Frausto; } \\
\text { Francisco Javier Gutiérrez Piña, Juan Antonio Ramírez } \\
\text { Chequer. }\end{array}$ \\
\hline Validación & $\begin{array}{l}\text { Fabiola Lydie Rochin Berumen; Heriberto Rodríguez Frausto; } \\
\text { Francisco Javier Gutiérrez Piña, Juan Antonio Ramírez } \\
\text { Chequer. }\end{array}$ \\
\hline Análisis Formal & Fabiola Lydie Rochin Berumen; Heriberto Rodríguez Frausto \\
\hline Investigación & Fabiola Lydie Rochin Berumen; Heriberto Rodríguez Frausto \\
\hline Recursos & $\begin{array}{l}\text { Fabiola Lydie Rochin Berumen; Heriberto Rodríguez Frausto; } \\
\text { Francisco Javier Gutiérrez Piña, Juan Antonio Ramírez } \\
\text { Chequer. }\end{array}$ \\
\hline Curación de datos & $\begin{array}{l}\text { Francisco Javier Gutiérrez Piña, Juan Antonio Ramírez } \\
\text { Chequer. }\end{array}$ \\
\hline $\begin{array}{l}\text { Escritura - Preparación del } \\
\text { borrador original }\end{array}$ & Fabiola Lydie Rochin Berumen; Heriberto Rodríguez Frausto \\
\hline $\begin{array}{l}\text { Escritura - Revisión y } \\
\text { edición }\end{array}$ & Fabiola Lydie Rochin Berumen; Heriberto Rodríguez Frausto \\
\hline Visualización & Heriberto Rodríguez Frausto \\
\hline Supervisión & Fabiola Lydie Rochin Berumen \\
\hline Administración de Proyectos & Fabiola Lydie Rochin Berumen \\
\hline Adquisición de fondos & $\begin{array}{l}\text { Fabiola Lydie Rochin Berumen; Heriberto Rodríguez Frausto; } \\
\text { Francisco Javier Gutiérrez Piña, Juan Antonio Ramírez } \\
\text { Chequer. }\end{array}$ \\
\hline
\end{tabular}

